\title{
Culture préscolaire et nouveaux défis au Japon
}

Pre-school culture and new challenges in Japan

Cultura preescolar y nuevos desafíos en Japón

\section{Miwako Hoshi Watanabe}

\section{OpenEdition}

\section{Journals}

Édition électronique

URL : http://journals.openedition.org/ries/899

DOI : $10.4000 /$ ries.899

ISSN : 2261-4265

Éditeur

Centre international d'études pédagogiques

Édition imprimée

Date de publication : 1 avril 2010

Pagination : 55-63

ISSN : 1254-4590

Référence électronique

Miwako Hoshi Watanabe, «Culture préscolaire et nouveaux défis au Japon », Revue internationale d'éducation de Sèvres [En ligne], 53 | avril 2010, mis en ligne le 01 avril 2013, consulté le 14 novembre 2019. URL : http://journals.openedition.org/ries/899; DOI : 10.4000/ries.899

(C) Tous droits réservés 


\section{Culture préscolaire et nouveaux défis au Japon}

\section{Miwako Hoshi Watanabe}

Au Japon, avant la scolarité obligatoire qui commence à l'âge de 6 ans, l'éducation préscolaire des enfants de 3 à 5 ans s'effectue dans le cadre d'un système dual. Ils peuvent fréquenter soit les jardins d'enfants (Yochi-en) qui sont sous la tutelle du ministère de l'éducation, soit les crèches collectives (Hoiku-en) qui sont sous la tutelle du ministère de la santé et du travail et accueillent jusqu'à l'âge de cinq ans les enfants dont les parents travaillent. Les jardins d'enfants privés et associatifs sont plus nombreux (61\%) que ceux qui sont publics (39\%). Les crèches publiques représentent $48 \%$ et les privées $52 \%$. Vont en crèche $40 \%$ des enfants de 5 ans.

Quant aux enfants de moins de 3 ans, $22 \%$ d'entre eux fréquentaient en 2009 les crèches, les autres étant pris en charge par les membres de la famille (y compris les grand-mères).

\section{LA CULTURE PRÉSCOLAIRE ENTRE TRADITION ET NOUVELLES ORIENTATIONS}

Dans chaque pays, les représentations culturelles et sociales de l'enfant fondent les conceptions de l'éducation préscolaire. L'image traditionnelle japonaise de l'enfant peut être résumée par le diction suivant : "Jusqu'à l'âge de 7 ans [selon le calendrier lunaire] l'enfant est dans la sphère des dieux ». C'est l'image d'un jeune enfant sain, naturel, vivant, libre et actif dans le jeu. La petite enfance se termine avec le septième anniversaire : l'enfant quitte alors le monde des dieux et entre dans la société des hommes, le monde des apprentissages. La distinction forte qui existe, au Japon, dans le système scolaire moderne, entre l'éducation primaire et l'éducation préscolaire n'est pas étrangère à cette représentation : il est évident que l'appellation, le diplôme de l'enseignant et le contenu de l'éducation préscolaire doivent être différents de ceux de l'école primaire.

L'esprit de l'éducation préscolaire japonaise doit beaucoup à Sozo Kurahashi (1882-1955), professeur en psychologie et pédagogie de la première école de formation des enseignantes du préscolaire ainsi que directeur du premier jardin d'enfants japonais. Sa théorie intègre les théories pédagogiques occidentales, principalement celles de Froebel et de Dewey, et les idées traditionnelles de l'éducation familiale fondées sur le respect de la bonne nature de l'enfant et 
l'offre de bons exemples de la part des adultes, et non sur une quelconque idée d'inculcation. Sa théorie est à la base du curriculum des jardins d'enfants, le Principe national de l'éducation préscolaire, qui cadre l'éducation préscolaire.

Selon sa théorie, développée principalement dans Éducation et la psychologie de l'enfant (1931, réédité en 1978), la petite enfance est une période unique et particulière dans laquelle les jeunes enfants passent des journées bien remplies, l'enfant devant être compris dans son existence globale et non comme l'assemblage de différents aspects. L'éducation des jeunes enfants ne vise pas l'acquisition de compétences, de techniques, de connaissances. Ce qui est le plus important dans la vie du jardin d'enfants est le jeu dans lequel toute la vie de l'enfant est exprimée et se trouve intégrée. Pendant que l'enfant joue en suivant ses initiatives et ses propres objectifs, il s'ouvre, déploie au maximum sa volonté et ses possibilités, et se développe. Le jeu peut survenir à n'importe quel moment. Le rôle de l'enseignant est alors de préparer l'environnement, d'inciter l'enfant au bon moment et ce, discrètement, pour que l'enfant s'oriente vers la direction favorable de lui-même, selon sa propre volonté. L'interaction avec les autres enfants est extrêmement importante pour stimuler les potentialités de l'enfant (Kurahashi, 1931).

\section{Un exemple de pédagogie du jeu}

Pour illustrer la pédagogie du jardin d'enfants japonais, voici un exemple d'activité, extrait de la documentation d'une enseignante de la classe des enfants de 5 ans d'un jardin d'enfants (Sato, 2009).

Un matin, une petite fille, K., apporte une bouteille dans laquelle il y a de l'eau et des zestes de citron. Elle dit qu'elle veut jouer au "marchand d'odeurs ». L'enseignante l'aide à installer le magasin. Les autres enfants collaborent à la fabrication d'un panneau. Les " clients » viennent. K., la « vendeuse ", approche la bouteille du nez de chaque client, qui sent. Un enfant lui demande de lui vendre de l'eau. K. lui répond: «Non, je ne peux pas vendre de l'eau. $\mathrm{Tu}$ peux sentir le parfum. ». Un autre enfant lui reproche : "Ce n'est pas un magasin, parce qu'il n'y a rien à vendre. ». Les enfants se disputent avec elle. L'enseignante intervient, en leur demandant de réfléchir à la façon de résoudre le problème. Les enfants donnent leur avis. Un enfant propose de vendre de l'eau dans des flacons. Les enfants se mettent d'accord. K., qui n'est pas d'accord au début, finit par accepter. L'enseignante donne des petits récipients dans lesquels les enfants mettent une petite quantité d'eau parfumée provenant de la bouteille. Ils sentent le parfum. Ensuite, ils développent leurs idées. Ils prennent des feuilles dans le jardin pour produire des parfums. L'enseignante multiplie les flacons. Ils mettent des étiquettes sur les flacons en écrivant le nom des feuilles. Le magasin a du succès. D'autres enfants viennent " acheter » les flacons. Mais à ce moment-là, K. commence à jouer seule. Ensuite, elle dit à l'enseignante : 
"Je vais jouer au jeu du magasin de l'écoute ». Et elle ouvre un " magasin », où les «clients» viennent parler et elle les écoute.

Le projet éducatif de ce jardin d'enfants repose sur trois étapes : " rencontrer », « rechercher », et " collaborer et créer », en favorisant les conduites telles que " sentir ", " réfléchir », " explorer », " interagir ", " écouter », " communiquer », " exprimer ». Dans ce cadre, l'enseignante a analysé cet épisode comme suit : K. a rencontré une expérience sensorielle qu'elle a voulu partager avec les autres enfants. Face au problème de partager quelque chose d'invisible, les enfants ont cherché une solution. Avec l'aide de l'enseignante, ils ont réfléchi, se sont exprimés, ont écouté les opinions des uns et des autres, cherché et collaboré. Ils sont parvenus à partager en inventant la parfumerie. Sur le plan cognitif, ils ont acquis des connaissances sur les odeurs et le nom de plantes. L'intervention de l'enseignante a été efficace dans le déroulement du jeu et ses transformations inattendues donnant lieu à une expérience riche. En produisant cette documentation, l'enseignante a découvert que l'intention de K. était de partager quelque chose à sentir, quelque chose d'invisible mais de précieux, alors qu'elle l'avait visualisé en introduisant des flacons. Et c'est pour cela que K. s'est tournée vers un autre jeu lié à une sensation invisible, le jeu de l'écoute. Partager ce qui est invisible mais existe est une expérience riche pour les enfants ; l'adulte, pense cette enseignante, doit être prêt à envisager de multiples possibilités de les aider, puisque les enfants ont un grand potentiel imaginatif.

\section{Le Principe national de l'éducation au jardin d'enfants}

Au Japon, les jardins d'enfants privés et associatifs ont plus de liberté et présentent donc une plus grande diversité de contenus éducatifs. Ils fonctionnent toutefois sur une base légale commune avec les jardins d'enfants publics, le Principe national de l'éducation au jardin d'enfants. Le premier texte (1948) se fonde sur la théorie de Kurahashi qui avait lui-même contribué à l'élaboration de ce curriculum. Depuis lors, nous retrouvons, en dépit de cinq révisions du texte $(1954,1968,1989,1998$ et 2008), ces mêmes propos : «L'éducation au jardin d'enfants doit se faire à travers l'aménagement de l'environnement, en respectant la spécificité de la petite enfance »; "L'éducation doit tenir compte des particularités de chaque enfant "; "Il faut encourager les enfants à prendre l'initiative de leurs activités »; "Les objectifs de l'éducation sont à atteindre à travers les jeux ».

Cela étant, le contenu du Principe national centré sur une éducation qui valorise entièrement le jeu et les particularités de la petite enfance, s'oriente aujourd'hui vers une préparation à l'école, mais sans prendre de forme scolaire (Hoshi-Watanabe, 1999). Les versions de 1989 et de 1999 soulignent l'importance du jeu et de la motivation. On y trouve les expressions : " éprouver de la 
joie », «s'intéresser » ou "réfléchir à sa façon », et non celles d' « acquérir» ou de "réussir ». Dans un contexte où, depuis quelques années, le ministère de l'éducation indique une augmentation des troubles causés par des écoliers qui dérangent les autres enfants, en première année de l'école primaire, faisant craindre une baisse des compétences scolaires, le passage du préscolaire au scolaire est discuté, avec l'idée que les enfants doivent être prêts à apprendre au moment de l'entrée à l'école primaire. Dans le cadre de la réforme de 2008, la Loi de l'éducation scolaire (loi fondamentale sur les écoles et les jardins d'enfants) redéfinit alors le jardin d'enfants comme une "institution qui cultive les bases de ce qui est enseigné à l'école obligatoire et ultérieurement " (art. 22). La réforme du Principe suit la loi dans cette direction. Ainsi, les objectifs spécifiques de l'éducation des jeunes enfants font place à l'objectif de préparation à l'école primaire. On saura les effets de cette évolution dans quelques années.

\section{Les obstacles à surmonter}

Il n'est pas étonnant, dans la société actuelle, que l'on s'oriente davantage vers l'éducation aux compétences intellectuelles. Outre cette pression sociale, il nous semble que cette "éducation centrée sur le jeu ", holistique", contient des facteurs internes l'empêchant de se développer. Nous en soulignerons trois.

Tout d'abord, il existe peu de recherches sur les effets du type d'éducation préscolaire sur la vie scolaire future. Nous ne disposons pas d'assez de preuves de l'efficacité de l'une ou autre approche. Jusqu'à présent, aucune étude longitudinale ni de comparaison n'a été effectuée au Japon.

Ensuite, les pratiques de "l'éducation centrée sur le jeu » exigent une grande compétence de la part des enseignants. Selon Tsumori (1980), l'enseignant doit être attentif, sans préjugé, à ce qui déroule chez l'enfant, et décoder ses petits gestes et expressions en interagissant avec lui. Il doit répondre de façon réfléchie et évaluer les effets de sa conduite. Tous les jours, il rencontre, auprès des enfants, de nombreux événements inattendus dont il doit trouver les significations, afin d'essayer de transformer ces rencontres en expériences de valeur. Il arrive souvent qu'il réagisse à l'enfant dans l'immédiat, au niveau sensoriel, dans la proximité du corps à corps. Dans ce cas, il doit y réfléchir et se documenter pour développer une compréhension plus approfondie. Ainsi, travailler au jardin d'enfants requiert réflexion, sensibilité, compétences communicatives et souplesse. L'enseignant devrait également se perfectionner dans les diverses techniques d'enseignement tout au long de sa vie professionnelle : observation, communication, documentation, etc. Pour assurer un certain niveau de qualité

\footnotetext{
1. Rapport Petite enfance, grand défi ll (OCDE, 2006) : «Dans l'approche holistique, tous les domaines de développement sont abordés à travers le jeu et dans le cadre d'un travail général sous forme de projets qui encourage l'apprentissage actif et la multiplication des expériences dans les principaux domaines de développement. Avec l'aide d'enseignants expérimentés, les jeunes enfants peuvent choisir leurs activités et organiser les projets » (p. 153).
} 
chez les enseignants inexpérimentés, non seulement la transmission des « virtuoses » aux novices est indispensable, mais aussi l'invention de moyens destinés à faciliter la maîtrise de ces techniques, faute de quoi les jeunes enseignants pencheront vers l'apprentissage de compétence prédéfinies dont les techniques d'enseignement sont plus faciles à maîtriser.

Enfin se pose le problème du statut des professionnels de la petite enfance au Japon. Le statut social et les conditions de travail de l'enseignant au jardin d'enfants sont inférieurs à ceux de l'enseignant de l'école, ce qui limite le pouvoir de développement de l'éducation préscolaire. En raison du faible salaire dans les jardins d'enfants privés et associatifs, les jeunes enseignantes interrompent souvent leur activité professionnelle après trois ou quatre années, avant de maîtriser les compétences évoquées pour être expert de l'éducation centrée sur le jeu. Par conséquent, il est probable que les novices mettent en œuvre des pratiques plus faciles, en donnant par exemple aux enfants des activités selon un programme prédéfini. L'essentiel, pour que l'éducation holistique se diffuse largement, c'est que la profession soit valorisée, avec des salaires et des conditions de travail réglementairement analogues à celles du jardin d'enfants et de l'école.

Si l'éducation des jeunes enfants est considérée comme la formation des fondements de la personnalité, son objectif est de contribuer à enrichir la vie future : pas uniquement la vie professionnelle ou économique, mais tous les aspects de la vie et pour toute la durée de la vie. Dans cette optique, la motivation, la sensibilité, les expressions corporelles et verbales, la relation avec autrui sont aussi importantes que la prévention de l'échec scolaire dans le futur proche. Les conséquences de la mondialisation, les effets désastreux de la politique néolibérale (augmentation de la pauvreté, perte de confiance, augmentation du taux de suicides et de dépressions, etc.) montrent que la qualité de la vie est plus précieuse que la compétition économique. Une éducation holistique de la petite enfance demeure à défendre davantage dans cette perspective, à l'avenir.

\section{QUelle ÉQuité Éducative ? ÉVOLUTIONS RÉCENTES}

L'idée essentielle sur laquelle repose la politique éducative du parti conservateur qui est resté au pouvoir cinquante ans, après la Deuxième Guerre mondiale, est que l'éducation est l'affaire des parents. Élever les enfants est une affaire privée qui ne relève pas de la société, ce n'est pas un investissement social. Une telle conception impose aux parents une lourde charge morale (il appartient aux parents d'élever de "bons » enfants, les parents sont responsables des problèmes de leurs enfants), sociale et économique (les parents sont responsables de la formation de leurs enfants pour qu'ils puissent gagner leur vie dans l'avenir). 
Les parents, de leur côté, trouvent en général naturel de se charger de l'éducation de leurs enfants et y investissent une grande partie de leurs revenus, ce qui était possible pour la plupart des familles dans les années quatre-vingts dans une société relativement homogène, mais ne l'est plus aujourd'hui.

Le gouvernement japonais investit faiblement dans l'éducation, vue comme un domaine privé. Selon l'OCDE (2009), l'investissement national du gouvernement japonais pour les enfants de 0 à 5 ans est de 13,261 dollars américains, ce qui correspond au quart de la dépense de la France et place le Japon au $24^{\mathrm{e}}$ rang sur les 29 pays concernés par l'étude. Il lui est aussi recommandé de dépenser considérablement plus pour les jeunes enfants afin de leur assurer un bon départ dans la vie.

L'investissement total du Japon dans l'éducation est de 3,3\% du PIB (moyenne des pays de l'OCDE : 4,9\%). La proportion de la dépense éducative par rapport à la dépense générale du gouvernement est de 9,5\%, bien inférieure aux 14,9\% correspondant à la moyenne de l'OCDE. La part de la charge familiale dans la dépense éducative est de $21,8 \%$, ce qui place le Japon à la deuxième place des pays concernés. La part pour l'éducation préscolaire s'élève à 38,3\% (OCDE, 2008).

Depuis les années quatre-vingt-dix, les différences entre classes sociales se sont considérablement accrues avec la politique néolibérale du gouvernement. Le nombre de familles qui ne peuvent pas payer les frais d'éducation a rapidement augmenté. Le gouvernement a, en outre, alourdi la charge des familles dans différents domaines de la sécurité sociale et du bien-être social.

Depuis 2000 environ, sont apparues de façon significative des inégalités éducatives selon les revenus des parents, y compris au niveau de la scolarité obligatoire et de l'accès à l'enseignement supérieur (Yuzawa et al. 2009). La scolarité obligatoire, tout en étant gratuite, exige des frais considérables (déjeuners, trajet scolaire) que certains parents ne peuvent aujourd'hui plus assumer. La prise de conscience de cette réalité est très récente, la tendance générale étant aux reproches adressés aux parents "irresponsables».

L'éducation préscolaire est onéreuse au Japon. Ainsi, les parents assument plus de $56 \%$ des frais dans les jardins d'enfants privés et associatifs ( $80 \%$ des enfants de 5 ans accueillis dans les jardins d'enfants le sont dans ce secteur privé et associatif). Les parents reçoivent des subventions dont un tiers vient de l'État et le reste de la collectivité locale. Cette somme varie, selon les collectivités, selon plusieurs variables : privé-public, revenus des parents, nombre d'enfants. Si pour certains parents, la somme payée est remboursée grâce à ces subventions, pour la majorité, ces dernières ne correspondent qu'à une petite partie de la somme dont ils doivent s'acquitter. Le Japon fait exception de ce point de vue, comparé à la majorité des pays membres de l'OCDE, où les frais pour l'éducation préscolaire sont gratuits pour les enfants de 3 à 5 ans. 


\section{La pauvreté des enfants}

Depuis dix ans, les inégalités sociales s'accroissent donc rapidement et le nombre d'enfants de familles pauvres augmente. L'OCDE (2009) constate que le taux de pauvreté des adultes s'élève à 15,7\% et celui des enfants seuls atteint $13 \%$ (moyenne OCDE : 12,4 \% ; France : 7,6 \%). Il en résulte une chaîne négative dans laquelle les enfants des familles pauvres ne peuvent pas recevoir assez d'éducation et ne peuvent trouver qu'un travail avec de faibles revenus. La dépense sociale dans le domaine familial est $0,75 \%$ au Japon, ce qui est bien inférieur aux 3,02 \% de la France (chiffres de 2006).

Le gouvernement japonais a longtemps fermé les yeux en ne recensant pas le taux de pauvreté. L'accroissement des inégalités met pourtant en évidence la pauvreté de certains enfants dans les écoles et les institutions de la petite enfance. La pauvreté dans les familles monoparentales est considérable (66\%). Le taux de pauvreté après impôts est plus haut que celui avant impôts. Cela montre que les familles pauvres deviennent plus pauvres par les systèmes d'impôts et de sécurité sociale qui sont moins favorables pour les familles pauvres que pour les familles aisées.

La dépression des parents, la maltraitance, l'effondrement des structures et des valeurs familiales traditionnelles ont augmenté (Yuzawa et al., 2009). Le soutien parental fait partie aujourd'hui de la mission des crèches, institutions du bien-être, et des écoles, lieu d'éducation obligatoire, mais pas de celle des jardins d'enfants.

\section{Vers la correction des inégalités?}

Ces problèmes liés à la pauvreté des enfants font l'objet de débats depuis 2008 et commencent à être reconnus. L'illusion que $80 \%$ des familles japonaises sont de classe moyenne s'est écroulée et de grandes inégalités deviennent évidentes. En août 2009, les Japonais ont voté pour une alternance politique. Ils s'attendent à de nouvelles politiques qui prennent en charge ce qui a été considéré comme relevant de la responsabilité des individus. Ils souhaitent un changement qui fasse passer de : "Il faut que les parents s'occupent de leurs enfants » à : "Il faut que la société soutienne les parents et les enfants ». En octobre 2009, le nouveau gouvernement, après avoir effectué une enquête, a publié un taux de pauvreté de $14,2 \%$ et s'est donné comme tâche importante de le faire baisser.

\section{Quel ACCuEIL DE LA DiVersité ?}

Jusqu'à maintenant, les politiques présupposaient un modèle familial traditionnel, dans lequel le père travaille à plein temps et la mère reste au foyer avec deux enfants. Les systèmes d'impôts, de sécurité sociale et d'assurance 
sociale prennent comme standard les familles correspondant à ce modèle. Les autres en bénéficient moins, étant alors qualifiées de familles « exceptionnelles » ou « déviantes ». Or les familles standard ne sont en réalité plus majoritaires, le gouvernement ayant conservé jusqu'ici ce modèle considéré comme souhaitable pour maintenir une structure sociale stable. Les politiques familiales japonaises se basent donc sur un modèle qui ne reconnaît pas la diversité actuelle des structures familiales.

Dans ce contexte de politiques publiques niant la diversité, l'habitus japonais est peu ouvert aux diversités culturelles et ethniques; il configure une manière de penser qui présuppose une société homogène. D’où la nécessité urgente d'une formation et d'une éducation aux diversités dès le préscolaire. Ce sont surtout des bénévoles qui travaillent activement dans cette direction dans les domaines de l'assistance sociale et de l'éducation scolaire.

Le Japon n'est pas un pays multiculturel ou multiethnique, à la différence d'autres pays. Sa population se compose d'une grande majorité japonaise, de minorités ethniques (dont les Ainu), de populations d'origine coréenne et chinoise avec leurs identités historiques, et de récents migrants venus d'Asie et d'Amérique du Sud. C'est depuis l'apparition de problèmes rencontrés par des familles migrantes (inadaptation à la société japonaise, conflits de voisinage, etc.) que se développent des discussions sur le multiculturalisme.

Les crèches et les jardins d'enfants, comme les écoles, se heurtent à un certain nombre de problèmes non résolus et, dans la majorité des cas, ce sont les éducateurs de ces institutions qui s'en chargent eux-mêmes. Il peut y avoir, par exemple, une incompréhension des parents concernant la crèche, une communication insuffisante avec les parents, des difficultés pour les enfants à vivre au jardin d'enfants, etc. Des bénévoles peuvent venir les aider. La formation continue, planifiée par les autorités locales, comporte peu de programmes sur le multiculturalisme, sauf dans les secteurs où les nouveaux arrivants sont nombreux. Par contre, les écoles ont créé des cours d' «éducation pour la compréhension internationale » et enseignent le japonais aux enfants dont la langue maternelle n'est pas cette langue, mais ces enfants n'ont pas d'occasions d'apprendre leur langue maternelle hors de chez eux. Au-delà des efforts individuels de certains, il manque un débat systématique sur la façon d'approfondir la compréhension du multiculturalisme.

Quant aux enfants handicapés, la situation n'est pas nouvelle et la réalité est différente. Ils sont systématiquement accueillis dans les crèches qui sont les institutions du bien-être des enfants. Le personnel est augmenté si nécessaire. Dans certaines collectivités, les psychologues font le tour des crèches et conseillent les équipes. Il existe également des stages pour les éducatrices. Pour les enfants handicapés, les crèches sont des lieux importants de soin et d'éducation pour grandir et se socialiser. Dans les jardins d'enfants, l'accueil des 
enfants handicapés dépend de chaque institution, car les jardins d'enfants ont la liberté de leur éducation et du choix des enfants.

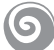

En tant que premier stade de l'éducation, qui dure toute la vie, l'éducation préscolaire se voit aujourd'hui un peu partout assigner un rôle plus important qu'auparavant, afin de privilégier l'apprentissage qui prépare à l'école.

De nombreux professionnels du secteur, ainsi que les auteurs du rapport Petite enfance, grand défi II (OCDE, 2006) recommandent au contraire une " approche holistique ", telle qu'elle est pratiquée dans les pays du Nord de l'Europe ou encore à Reggio Emilia, en Italie.

Ces propositions et recommandations sont encourageantes pour l'éducation préscolaire japonaise, car celle-ci se situe historiquement du côté holistique, et non du côté de la préparation à l'école. Loin d'être démodée, elle se voit ainsi reconnue et valorisée. Cependant, au Japon aussi, l'éducation holistique traditionnelle est menacée.

\section{BIBLIOGRAPHIE}

DAHLBERG G. et MOSS P. (2007) : "Au-delà de la qualité, vers l'éthique et la politique en matière d'éducation préscolaire ». In Brougère, G. et Vandenbroeck, M. (dir.) Repenser l'éducation des jeunes enfants. Bruxelles : Peter Lang, p. 53-76.

HOSHI-WATANABE M. (1999) : «Play or Learn? Inside and Outside of Preschool in Changing Japanese Society ». In Brougère, G. \& Rayna, S. (dir.). Culture, Childhood and Preschool Education. Paris : UNESCO, p. 85-99.

OCDE (2006) : Petite enfance, grand défi II. Paris : OCDE.

OCDE (2008) : Inégalités croissantes? Distribution des revenus et pauvreté dans les pays de l'OCDE. Paris: OCDE.

OCDE (2009) : Assurer le bien-être des enfants. Paris : OCDE.

SATO H. (2009) : "Partager ce qui n'est pas visible ». L'éducation des jeunes enfants, 108, 5, p. 52-67 (en japonais).

TSUMORI M. (1980) : Expériences et réflexions sur l'éducation des jeunes enfants. Tokyo : Editions Dainihon-Tosho (en japonais).

YUZAWA N. et al. (2009) : Papier blanc sur la pauvreté des enfants. Tokyo : Editions Akashi (en japonais).

KURAHASHI S. (1931) : Education et la psychologie de l'enfant. Réédité en 1978. Editions Japan Library, collection " Documents sur l'éducation préscolaire à l'époque de Taisho et de Showa », p. 65-164 (en japonais). 
